\title{
241. Quelques exemples des ל-fonctions d'Epstein pour les opérateurs elliptiques dégénérés du second ordre. II
}

\author{
Par Norio SHIMAKURA
}

(Comm. by Kinjirô Kunugi, M. J. A., Dec. 12, 1970)

Ce petit mémoire est la suite de mon article précédent [4]. Premièrement, dans le paragraphe 1 de [4], le Théorème 1 et la preuve du Théorème 2 contenaient des fautes (le Théorème 2 lui-même a été sauvé). Je vais les corriger dans le paragraphe 4. Et le paragraphe 5 est consacré à citer un autre exemple simple des $\zeta$-fonctions d'Epstein.

$\S 4$. Je vais reproduire tous les contenus du $\S 1$ dans [4]. Soient $n$ un entier $\geqq 2$ et $\Omega$ la boule unitaire de $\boldsymbol{R}^{n}$ :

$$
\Omega=\left\{x=\left(x_{1}, \cdots, x_{n}\right) \in \boldsymbol{R}^{n} ;|x| \equiv\left(\sum_{j=1}^{n} x_{j}^{2}\right)^{1 / 2}<1\right\} .
$$

Traitons, dans $\Omega$, un opérateur différentiel $L$ elliptique dégénéré au bord

$$
L u(x)=-\sum_{j=1}^{n} \frac{\partial}{\partial x_{j}}\left\{\left(1-|x|^{2}\right) \frac{\partial u}{\partial x_{j}}(x)\right\}+(n-1) u(x) .
$$

$L$ est auto-adjoint positif dans $L^{2}(\Omega)$ du domaine $\mathscr{D}[L]=\left\{u(x) \in H^{1}(\Omega)\right.$; $\left.\left(1-|x|^{2}\right) u(x) \in H^{2}(\Omega)\right\}$. Son spectre se consiste des valeurs propres positives $\left\{\lambda_{k, l}\right\}_{k, l=0}^{\infty}$ dont chacune $\lambda_{k, l}$ est de multiplicité $\mu(k)<\infty$, où

$$
\begin{gathered}
\lambda_{k, l}=(2 l+2)(2 l+2 k+n-1), \text { pour } k, l=0,1,2, \cdots ; \\
\left\{\begin{array}{l}
\mu(0)=1 \text { et } \mu(k)=2 \text { pour } k \geqq 1 \text {, si } n=2 ; \\
\mu(k)=(2 k+n-2) \frac{(k+n-3) !}{k !(n-2) !} \text { pour } k \geqq 0, \text { si } n \geqq 3 .
\end{array}\right\}
\end{gathered}
$$

Proposition 1. ( $\alpha$ ) $\left\{\lambda_{k, l}\right\}_{k, l=0}^{\infty}$ est la totalité des valeurs propres de $L$ dont chacune $\lambda_{k, l}$ est de multiplicité $\mu(k)<\infty$;

( $\beta) \operatorname{Pour}(k, l)$ fixe, une base des fonctions propres correspondante à $\lambda_{k, l}$ est formée par les $\mu(k)$ fonctions (normalisées) suivantes

$$
\begin{gathered}
u_{k, l, \alpha}(x)=\sqrt{4 l+2 k+n} P_{l}^{(0, k+\nu)}\left(2|x|^{2}-1\right) H_{k, \alpha}(x), \\
\text { pour } k, l=0,1,2, \cdots ; 1 \leqq \alpha \leqq \mu(k),
\end{gathered}
$$

où $\left\{H_{k, \alpha}(x)\right\}_{\alpha=1}^{\mu(k)}$ est une base des polynômes harmoniques homogènes d'ordre $k$, les $P_{l}^{(a, b)}(t)$ sont les polynômes de Jacobi (voir p. $168 d u$ Vol. 2 de [2]), et dernièrement $\nu=(n-2) / 2$.

Soit maintenant $T>0$ très grand et désignons par $N(T)$ la somme des $\mu(k)$ pour lesquelles $\lambda_{k, l} \leqq T$. Alors, Théorème 1 dans [4] se corrige comme suit:

Theoreme 1. Lorsque $T$ tend vers l'infini, $N(T)$ se comporte asymptotiquement comme suit: 


$$
\begin{aligned}
& N(T) \sim \frac{T}{4} \log T \quad, \text { si } n=2 ; \\
& N(T) \sim \frac{2^{3-2 n} \zeta(n-1 ; 1 / 2)}{(n-1) !} T^{n-1}, \text { si } n \geqq 3 .
\end{aligned}
$$

Démonstration de (6). Soit $T>0$ suffisamment grand et notons par $\nu(l, T)$, pour $l$ fixe, le nombre des $k$ pour lesquels $\lambda_{k, l} \leqq T$. Alors, en posant $T_{1}=(\sqrt{T}-1) / 2$, nous avons

$$
\left\{\begin{array}{l}
\nu(l, T)=\left[\frac{T}{2(2 l+1)}-l+\frac{1}{2}\right], \\
\text { et } \nu(l, T)=0 \leqq l \leqq\left[T_{1}\right],
\end{array}\right.
$$

où $\left[T_{1}\right]$ est la partie entière de $T_{1}$, et nous avons naturellement

$$
N(T)=\nu(0, T)+2 \sum_{l=1}^{\left[T_{1}\right]} \nu(l, T) .
$$

Estimons la différence $N(T)-\nu(0, T)$ :

$$
\begin{aligned}
& 2 \int_{0}^{T_{1}}\left(\frac{T}{2(2 t+1)}-t-\frac{1}{2}\right) d t<N(T)-\nu(0, T) \\
& \quad \leqq 2 \int_{0}^{T_{1}}\left(\frac{T}{2(2 t+1)}-t+\frac{1}{2}\right) d t+\frac{T+1}{2} .
\end{aligned}
$$

Le calcul de ces intégrales est facile et nous obtenons finalement (6), car $\nu(0, T) \sim T / 2$.

Démonstration de (7). Nous pourrions vérifier (7) par le même principe que celui du cas $n=2$ ci-dessus, mais il sera intéressant d'introduire la $\zeta$-fonction d'Epstein pour $L$

$$
\zeta_{L}(s)=\sum_{k=0}^{\infty} \sum_{l=0}^{\infty} \mu(k) \lambda_{k, l}^{-s},
$$

et de déduire (7) par la recherche des singularités de $\zeta_{L}(s)$ dans le $s$-plan complexe. Remarquons que la série de (10) est absolument convergente si Re. $s>n-1$ grâce au résultat de Baouendi-Goulaouic (voir le Théorème 4 de [1]). Nous allons maintenant extraire toutes les singularités possibles de $\zeta_{L}(s)$ et démontrer qu'elle est une fonction méromorphe dans le $s$-plan tout entier. Pour l'effectuer, nous avons besoin d'un lemme:

Lemme. Soient $x>0, y>0$ et posons, pour Re. $\beta>1$ et Re. $(\alpha+\beta)$ $>2$,

$$
\left\{\begin{array}{l}
F(\alpha, \beta ; x, y)=\sum_{l=0}^{\infty}(l+x)^{-\alpha} \sum_{p=l}^{\infty}(p+y)^{-\beta}, e t \\
f(\alpha, \beta ; x, y)=\sum_{l=0}^{\infty}(l+x)^{-\alpha} \int_{l}^{\infty}(v+y)^{-\beta} d v .
\end{array}\right\}
$$

Lorsqu'on fixe $x>0$ et $y>0$,

(i) la fonction $(\beta-1) f(\alpha, \beta ; x, y) / \Gamma(\alpha+\beta-2)$ se prolonge comme une fonction entière de $(\alpha, \beta)$; et,

(ii) la fonction $F(\alpha, \beta: x, y) /\{\Gamma(\beta-1) \Gamma(\alpha+\beta-2)\}$ le fait également. 
Preuve. $F$ et $f$ satisfont à l'équation fonctionnelle

$$
\frac{\partial}{\partial y} u(\alpha, \beta ; x, y)=-\beta u(\alpha, \beta+1 ; x, y),
$$

et, lorsque $y=x>0$, nous voyons que

$$
f(\alpha, \beta ; x, y)=\frac{1}{\beta-1} \zeta(\alpha+\beta-1 ; x),
$$

où $\zeta(z ; a)=\sum_{k=0}^{\infty}(k+a)^{-z}$ (Re. $z>1$ et $\left.a>0\right)$ est la $\zeta$-fonction de Riemann. D'où l'on a le développement, pour chaque $k$ : entier $\geqq 1$,

$$
\begin{aligned}
f(\alpha, \beta ; x, y)= & \sum_{j=0}^{k-1} \frac{\Gamma(\beta+j-1)}{\Gamma(\beta) j !}(x-y)^{j \zeta}(\alpha+\beta+j-1 ; x) \\
& -\frac{\Gamma(\beta+k)}{\Gamma(\beta)(k-1) !} \int_{x}^{y}(u-y)^{k-1} f(\alpha, \beta+k ; x, u) d u .
\end{aligned}
$$

Dans le membre à droite de (14), le dernier terme est holomorphe dans Re. $\beta>1-k$ et Re. $(\alpha+\beta)>2-k$. Nous avons donc extrait, comme la première somme à droite de (14), toutes les singularités de $f$ situées dans Re. $\beta>1-k$ et Re. $(\alpha+\beta)>2-k$, et nous connaissons que la fonction $\zeta(z ; a)-1 /(z-1)$ est une fonction entière de $z$, d'où l'énoncé (i) $d u$ lemme.

Passons ensuite à la recherche de $F$ : Nous voyons d'abord que

$$
f(\alpha, \beta ; x, y)=\int_{0}^{1} F(\alpha, \beta ; x, y+u) d u \text {. }
$$

Posons $G(\alpha, \beta ; x, y)=F(\alpha, \beta ; x, y)-f(\alpha, \beta ; x, y)$. Alors, nous avons

$$
\begin{aligned}
G(\alpha, \beta ; x, y)= & \beta \int_{0}^{1} F(\alpha, \beta+1 ; x, y+1-u) u d u \\
= & \sum_{j=1}^{k} \frac{\Gamma(\beta+j)}{\Gamma(\beta)} \int_{0}^{1} \cdots \int_{0}^{1} f(\alpha, \beta+j ; x, y \\
& \left.+j-u_{1}-\cdots-u_{j}\right) u_{1} \cdots u_{j} d u_{1} \cdots d u_{j} \\
& +\frac{\Gamma(\beta+K)}{\Gamma(\beta)} \int_{0}^{1} \cdots \int_{0}^{1} G(\alpha, \beta+k ; x, y \\
& \left.+k-u_{1}-\cdots-u_{k}\right) u_{1} \cdots u_{k} d u_{1} \cdots d u_{k}, \\
& \text { quel que soit } k: \text { entier } \geqq 1 .
\end{aligned}
$$

La première égalité de (16) nous montre que $G(\alpha, \beta ; x, y)$ est holomorphe dans Re. $\beta>0$ et Re. $(\alpha+\beta)>1$. Et la deuxième égalité extrait, comme la première somme à droite, toutes les singularités situées dans Re. $\beta>-k$ et Re. $(\alpha+\beta)>1-k$. Nous avons enfin l'énoncé (ii) grâce à (i).

C.Q.F.D.

Corollaire. Les fonctions

$$
f(\alpha, \beta ; x, y)-\frac{\zeta(\alpha+\beta-1 ; x)}{\beta-1} \text { et } F(\alpha, \beta ; x, y)-\frac{\zeta(\alpha+\beta-1 ; x)}{\beta-1}
$$

sont holomorphes dans Re. $\beta>0$ et Re. $(\alpha+\beta)>1$. 
Nous revenons à la fonction $\zeta_{L}(s)$. Exprimons-la à l'aide des $F(\alpha, \beta ; x, y)$ :

$$
\begin{aligned}
& \zeta_{L}(s)=2^{1-2 s} F\left(s, s ; \frac{1}{2}, \frac{1}{2}\right)-2^{-2 s \zeta}\left(2 s ; \frac{1}{2}\right), \text { si } n=2 ; \\
& 2^{2 s \zeta} \zeta_{L}(s)= \frac{2}{(n-2) !} \sum_{p=0}^{n-2}(-1)^{p}\left(\begin{array}{c}
n-2 \\
p
\end{array}\right) F\left(s-p, s+p+2-n ; \frac{1}{2}, \frac{n-1}{2}\right) \\
&+\sum_{q+r<n-2} C_{q, r} F\left(s-q, s-r ; \frac{1}{2}, \frac{n-1}{2}\right), \text { si } n \geqq 3,
\end{aligned}
$$

où les $C_{q, r}$ sont des constantes numériques indépendantes de $s$. Nous pouvons en déduire le résultat suivant:

Theoreme 2. Si $n \geqq 2, \zeta_{L}(s) /\{\Gamma(s-n+1) \Gamma(2 s-n)\}$ se prolonge comme une fonction entière de $s$;

(ii) $\operatorname{Sin}=2,4(s-1) \zeta_{L}(s)-(s-1)^{-1}$ est holomorphe dans Re. $s>\frac{1}{2}$; (iii) Si $n \geqq 3$,

$$
\zeta_{L}(s)-\frac{2^{3-2 n}}{(s-n+1)(n-2) !} \zeta\left(n-1 ; \frac{1}{2}\right)
$$

est holomorphe dans Re. $s>n-\frac{3}{2}$.

Fin de la démonstration de (7). On n'a qu'à appliquer le théorème tauberien d'Ikéhara à $\zeta_{L}(s)$ dont la singularité la plus à droite $s=n-1$ est pôle simple, et le résidu à ce point est connu par le (iii) du Théorème 2.

C.Q.F.D.

$\S 5$. Dans ce paragraphe, je vais citer un autre exemple de $\zeta$-fonction d'Epstein pour un opérateur elliptique.

Soit $M$ une variété riemannienne compacte sans bord de dimension $(n-1)$ dont le point générique est noté par $\xi$. Posons

$$
\mathcal{L} \phi(\xi)=\mathcal{L}\left(\xi ; \frac{\partial}{\partial \xi}\right) \phi(\xi)=\phi(\xi)-\Delta_{M} \phi(\xi),
$$

où $\Delta_{M}$ est l'opérateur de Laplace-Beltrami sur $M$. Alors, $\mathcal{L}$ est autoadjoint $\geqq I$ sur $L^{2}(M)$ par rapport à l'élément volumique $d \xi$ sur $M$, le domaine de $\mathcal{L}$ est $H^{2}(M)$. Soit $\left\{\mu_{j}\right\}_{j=1}$ la suite des valeurs propres de $\mathcal{L}$ numérotées suivant l'ordre de croissance : $1=\mu_{1} \leqq \mu_{2} \leqq \cdots$ (on répète chaque $\mu_{j}$ autant de fois que sa multiplicité). Désignons par $\left\{w_{j}(\xi)\right\}_{j=1}^{\infty}$ le système orthonormal complet formé par les fonctions propres $w_{j}(\xi)$ correspondantes à $\mu_{j}$. Considérons la variété $\mathcal{M}=M \times \boldsymbol{R}_{+}=M$ $\times\{t ; 0<t<\infty\}$ et traitons sur $\mathscr{M}$ un opérateur différentiel

$$
L u(\xi, t)=-\frac{\partial}{\partial t}\left(t \frac{\partial u}{\partial t}\right)+t \mathcal{L}\left(\xi ; \frac{\partial}{\partial \xi}\right) u(\xi, t)
$$

Alors $L$ est elliptique du second ordre qui est dégénéré sur le bord $\partial \mathscr{M}=M \times\{t=0\}$. Il est de plus auto-adjoint $\geqq I$ sur $L^{2}(\mathscr{M})$ du domaine $\mathscr{D}[L]=\left\{u(\xi, t) \in H^{1}(\mathscr{M}) ; t u(\xi, t) \in H^{2}(\mathscr{M})\right\}$. Son spectre se consiste des valeurs propres $\left\{\lambda_{l, j}\right\}_{l, j=1}^{\infty}$ dont chacune est de multiplicité 1 , où 


$$
\lambda_{l, j}=(2 l-1) \sqrt{\mu_{j}} \text {, pour } l, j=1,2, \cdots
$$

Pour chaque $(l, j)$, la fonction propre (normalisée) correspondant à $\lambda_{l, j}$ est

$$
u_{l, j}(\xi, t)=\left(4 \mu_{j}\right)^{1 / 4} w_{j}(\xi) \exp \left(-\sqrt{\mu_{j}} t\right) L_{l-1}\left(2 \sqrt{\mu_{j}} t\right),
$$

où les $L_{p}(z)=\frac{1}{p !} e^{z}\left(\frac{d}{d z}\right)^{p}\left(e^{-z} z^{p}\right)$ sont les polynômes de Laguerre.

Cela posé, la $\zeta$-fonction d'Epstein par rapport à $L$ se definit par

$$
\zeta_{L}(s)=\sum_{l, j=1}^{\infty} \lambda_{l . j}^{-s}, \text { pour Re. } s>n-1 .
$$

Nous pouvons la comparer avec la $\zeta$-fonction d'Epstein par rapport à $\mathcal{L} \operatorname{sur} M$ :

$$
\zeta_{M}(s)=\sum_{l, j=1}^{\infty} \mu_{j}^{-s}, \text { pour Re. } s>\frac{n-1}{2} .
$$

Nous obtenons une équation fonctionnelle entre elles

$$
\zeta_{L}(s)=2^{-s \zeta}\left(s ; \frac{1}{2}\right) \zeta_{M}\left(\frac{s}{2}\right), \text { pour Re. } s>n-1 .
$$

Il est connu (voir [3]) que le produit $\Gamma\left(s-\frac{n-1}{2}\right) \zeta_{M}(s)$ se prolonge comme une fonction entière de $s$. Par conséquent, le produit $(s-1)$ $\cdot \Gamma\left(\frac{s-n+1}{2}\right) \zeta_{L}(s)$ le fait également.

\section{Références}

[1] M. S. Baouendi et C. Goulaouic: Régularité et théorie spectrale pour une classe d'opérateurs elliptiques dégénérés. Archive for Rat. Mech. Anal., 34, 361-379 (1969).

[2] A. Erdélyi, W. Magnus, F. Oberhettinger, et F. G. Tricomi: Higher Transcendental Functions. McGraw-Hill.

[ 3 ] S. Minakshisundaram et A. Pleijel: Eigenfunctions of the Laplace operator on Riemannian Manifolds. Canadian J. Math., 242-256 (1949).

[4] N. Shimakura: Quelques exemples des $\zeta$-fonctions d'Epstein pour les opérateurs elliptiques dégénérés du second ordre. Proc. Japan Acad., 45(10), 866-871 (1969). 